\title{
Hibernisation und Strahlenschutz
}

\author{
HANS A. KüNKEL \\ Strablenbiologisches Institut der Universität Hamburg
}

\begin{abstract}
Hibernation and radiation protection. When hibernating loirs (Glis glis) are irradiated with lethal doses of $\mathrm{X}$-rays, radiation damage cannot be observed as long as the aninals are in the poikilothermic state. Only when they have returned to the homoeothermic state, does the typical radiation syndrom occur, and the animals die as if they were irradiated at the moment of awakening. The final rate of mortality, however, is about the same as in animals irradiated in the homoeothermic state. When the loirs (irradiated with $700 \mathrm{r}$ during hibernation) were kept in the hibernating state for 3 weeks and then injected with cysteine just at the moment of the awakening, $95 \%$ of the animals survived. This surprising aftereffect of cysteine was demonstrated in several typical biochemical reactions, e. $g$. in the rayinduced changes of the serum proteins by electrophoresis, in the production of erythrocytes by the incorporation of radioiron, in the DNA-synthesis, but also histologically in the cells of the bone marrow and of other organs. The possible mechanism of the protecting effect of cysteine applied 3 weeks after irradiation is discussed.
\end{abstract}

\section{EINLEITUNG}

Das Reizvolle an eirem solchen Symposion wie diesem ist zweifellos die Tatsache, daß es Wissenschaftler aus den verschiedensten Spezialgebieten zur Diskussion eines bestimmten Themas zusammenführt, und das ist hier die quantitative Biologie des Stoffwechsels. Die besonderen Schwierigkeiten liegen jedoch häufig darin, sich den Kollegen aus dem anderen Spezialgebiet verständlich zu machen, denn in der Wissenschaft ist es heutzutage of so wie beim Turmbau von Babylon, daß der eine die Sprache des anderen nicht mehr versteht. Gestatten Sie mir daher einige Vorbemerkungen, mit welchen ich denjenigen, denen die Wirkungen ionisierender Strahlen etwas verhältnismäßig Unbekanntes sind, kurz die Grundprobleme der Strahlenbiologie erläutere und einige Begriffe aus diesem Grenzgebiet zwischen Physik, Chemie, Biologie und Medizin definiere.

Die biologische Wirkung ionisierender Strahlen ist durch einige besondere charakteristische Merkmale gekennzeichnet, welche sie von anderen physikalischen Einwirkungen auf lebende Organismen grundlegend unterscheiden.

1. Bereits außerordentlich kleine Beträge absorbierter Strahlungsenergie können erhebliche biologische Wirkungen hervorrufen - im Extremfalle den Zelltod, die Zerstörung ganzer Organe oder gar den Tod eines komplizierten Organismus. So liegt $z$. B. die gesamte vom Körper absorbierte Energie einer tödlichen Röntgenbestrahlung um Größenordnungen niedriger als die uns von der Sonne in Form von Licht und Wärme 
zugeführte Energie, wenn wir im Sommer eine Stunde in der Sonne spazierengehen. Es würde z. B. die Wärmeenergie, die einem Menschen durch das Trinken einer Tasse Tee zugeführt wird, unweigerlich tödlich wirken, wenn sie ihm in Form von Röntgenstrahlen verabfolgt würde (Abb. 1).

2. Ein weiteres charakteristisches Merkmal der biologischen Strahlenwirkung dïrfte darin zu erblicken sein, daß es bislang nur in Ausnahmefällen gelungen ist, eine eimmal gesetzte massive Strahlenschädigung nachträglich durch physikalische oder
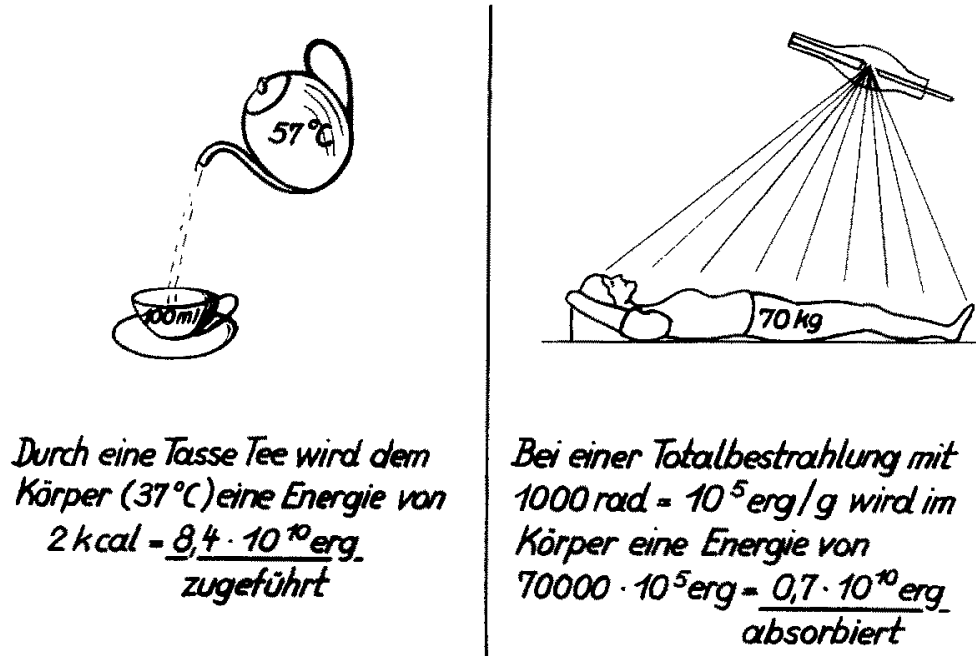

Abb. 1: Vergleich der absorbierten Energie einer Ganzkörperbestrahlung mit 1000 rad und der Energiezufuhr durch das Trinken einer Tasse Tee

chemische Einwirkungen wesentlich zu beeinflussen: Alle Versuche einer "Heilung“ eines starken Strahleninsults sind bis heute noch recht unbefriedigend geblieben und konnten sich zumeist nur auf eine Art „Substitutionstherapie ${ }^{\text {"t }}$ erstrecken. Auch die sogenannten chemischen Strahlenschutzsubstanzen zeigen normalerweise nur dann eine Wirkung, wenn sie vor der Bestrahlung appliziert werden. Diese Verbindungen müssen also während der Bestrahlung in den Körperzellen anwesend sein, um wirksam werden zu können. Das deutet darauf hin, daß die dem physikalischen Primärereignis der Strahlenabsorption folgenden strahleninduzierten biochemischen Reaktionsschritte in den meisten Fällen außerordentlich schnell ablaufen und sehr bald zu einem irreversiblen Zustand führen, in welchem der weitere Verlauf der nun folgenden Reaktionsketten nicht mehr wesentlich beeinflußt werden kann.

Es lag daher nahe, strahlenbiologische Untersuchungen unter solchen Bedingungen vorzunehmen, bei denen bereits der natïliche Ablauf der Lebensvorgänge erheblich verlangsamt ist, mit anderen Worten also, im Zustand reduzierten Stoffwechsels. Unter solchen Bedingungen kann vermutet werden, daß viele der interessierenden strahleninduzierten Prozesse ebenfalls quasi im Zeitlupentempo ablaufen oder unter Umständen sogar blockiert sind und somit einer experimentellen Beobachtung leichter zugänglich werden. Bei einigen biologischen Objekten kann eine solche Reduktion des 
Stoffwechsels leicht - ohne die "Lebenstüchtigkeit" zu beeinträchtigen - durch Erniedrigung der Umgebungstemperatur herbeigeführt werden. In der Klasse der Säugetiere erscheinen hierfür die natürlichen Winterschläfer besonders geeignet.

\section{DEFINITIONEN}

Bevor ich jedoch auf unsere eigenen Versuche eingehe, möchte ich noch einige Begriffe aus der Strahlenbiologie definieren, die mir gerade für das Problem Hibernisation und Strahlenschutz besonders wichtig erscheinen. Bestrahlt man ein Kollektiv von Versuchstieren mit einer genügend hohen Dosis ionisierender Strahlen, sagen wir mit einigen $100 \mathrm{rad}$, so wird ein großer Teil dieser Tiere binnen kurzem unter ziemlich komplexen Symptomen mehr oder weniger stark erkranken. Dieses Krankheitsbild bezeichnet man als "akutes Strahlensyndrom". Ein Teil der Tiere wird sich von dieser Strahlenkrankheit erholen und ein gewisser Prozentsatz wird an dem Strahlenschaden zugrundegehen. Man weiß aus zahlreichen Untersuchungen an den verschiedensten Versuchsobjekten, daß dieser "akute Strahlentod" innerhalb der ersten Wochen nach der Bestrahlung eintritt. Man hat daher vereinbart, bei allen Experimenten, in welchen die Mortalitätsrate nach Bestrahlung festgestellt werden soll, die Beobachtung auf einen Zeitraum von 30 Tagen zu beschränken. Alles was sich nach

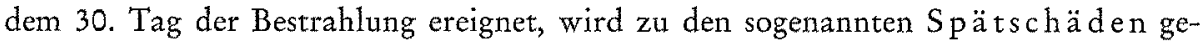
rechnet. Man spricht daher z. B. von einer "L $\mathrm{LD}_{50 / 30}$ " und meint damit diejenige Strahlendosis, bei welcher $50 \%$ der Tiere innerhalb von 30 Tagen sterben. Aufschlußreicher als die Angabe der $L_{50} / 30$ ist natürlich eine vollständige $\mathrm{D}$ os is ef $f \mathrm{ekt}-$ kurve, aus welcher die jeweilige Mortalitätsrate bei verschieden hohen Dosen zu ersehen ist. Wie ich schon andeutete, läßt sich durch Verabreichung bestimmter chemischer Verbindungen vor der Bestrahlung die Mortalitätsrate bis zu einem gewissen Grad reduzieren. Man spricht im Zusammenhang mit dem biologisch-chemischen Strahlenschutz von einem Dosis-Reduktionsfaktor, der allerdings keine konstante Größe ist, sondern mit der Höhe der Dosis sowie der Dosisleistung und selbstverständlich auch mit dem betreffenden biologischen Objekt und der geprüften Reaktion variiert. Man weiß ferner seit langem, daß der akute Strahlentod um so früher eintritt, je höher die applizierte Strahlendosis ist. Bei sehr hohen Dosen von einigen hunderttausend rad kann der Tod, wie RAJEwSKY et al. (1953a, b, 1954) gezeigt haben, in wenigen Minuten oder gar Sekunden nach der Bestrahlung erfolgen. Auch hier ist im allgemeinen die Aufstellung einer sogenannten Absterbeordnung aufschlußreicher als die Angabe einer mittleren Überlebenszeit. Die Absterbeordnung kann ebenfalls durch Verabfolgung von Strahlenschutzstoffen beeinflußt und die mittlere Überlebenszeit verlängert werden. Es scheint mir jedoch nicht sehr sinnvoll zu sein, von einem biologischen oder chemischen Strahlenschutz zu sprechen, wenn z. B. der Strahlentod lediglich um ein paar Tage verzögert wird, falls nicht gleichzeitig die endgültige Mortalitätsrate signifikant vermindert würde. 


\section{ERGEBNISSE}

Unsere eigenen Untersuchungen (zusammenfassende Darstellung: KünkeL \& SchUdert 1959, KüNkel 1961) wurden an Siebenschläfern (Glis glis) durchgeführt, einer vorwiegend in Süddeutschland vorkommenden Schlafmäuseart (Abb. 2). Diese Tiere lassen sich relativ leicht dadurch in den Winterschlaf versetzen, daß man sie ohne jede medikamentöse Beeinflussung in einen Kühlschrank setzt, der auf eine Tempera-

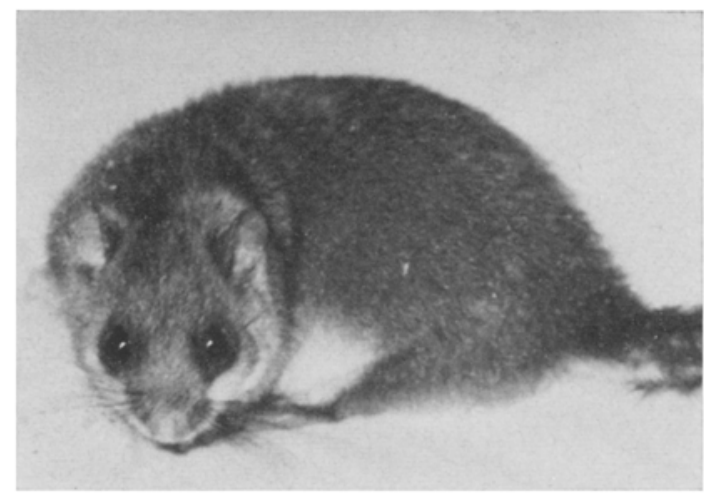

Abb. 2: Siebenschläfer (Glis glis)

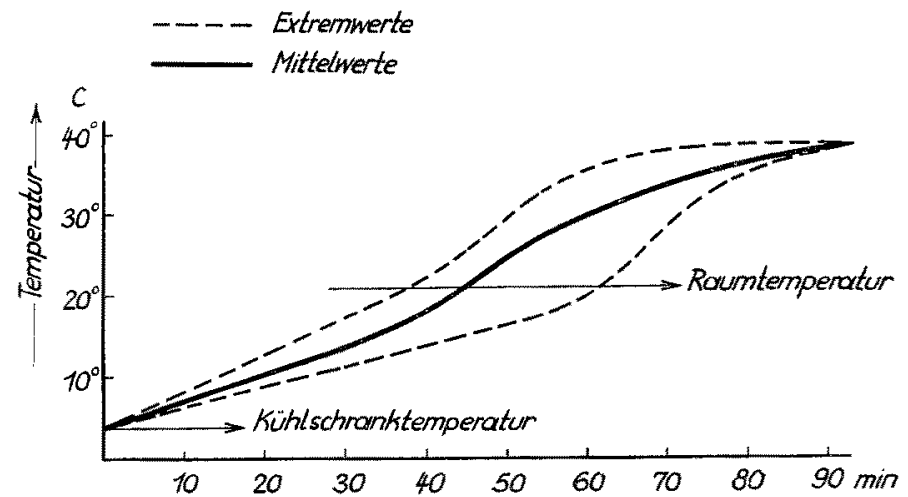

Abb. 3: Anstieg der Körpertemperatur von Siebenschläfern beim Erwachen aus dem Winterschlaf

tur zwischen $+2^{0}$ und $+4^{0} \mathrm{C}$ eingestellt ist. Nach wenigen Tagen beginnen sie dann zu schlafen. Bei höheren Temperaturen besteht die Gefahr, daß die Tiere zeitweilig erwachen; bei tieferen Temperaturen wird offenbar durch den Kältereiz eine Alarmreaktion inganggesetzt, und die Tiere wachen ebenfalls auf. Im Winterschlaf befinden sie sich in einem echten poikilothermen Zustand, das heißt, ihre Körpertemperatur ist exakt gleid der Umgebungstemperatur. Um die Siebenschläfer wieder in den homoiothermen Zustand zu versetzen, wurden sie in einen Raum mit Zimmertemperatur gebracht. Es dauert dann etwa 1 bis $1 \frac{1}{2}$ Stunden, bis die Tiere wieder völlig wach sind. 
Die Temperaturkurve, die in Abbildung 3 dargestellt ist, wurde mit Hilfe eines elektronischen Thermometers gewonnen.

$U_{m}$ Bestrahlungsexperimente im Winterschlaf vornehmen zu können, wurden die Siebenschläfer nach einer Woche Kühlschrankaufenthalt in einen speziell für diesen $Z_{\text {wedk }}$ konstruierten Thermostaten gebracht, der mittels eines Kühlaggregates ebenfalls auf einer konstanten Temperatur von $+2^{\circ} \mathrm{C}$ gehalten wurde, jedoch mit einer dünnen Plexiglasscheibe abgedeckt war, durch welche die Siebenschläfer einer Ganzkörperbestrahlung ausgesetzt werden konnten, ohne im Schlaf gestört zu werden (Abb. 4).

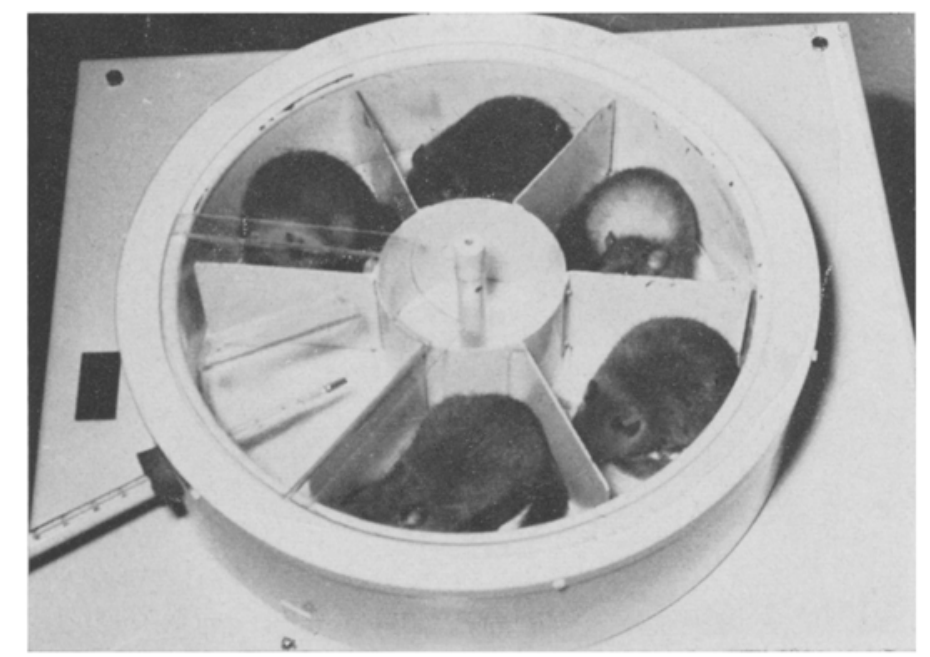

Abb. 4: Fahrbarer Thermostat zur Bestrahlung der Siebenschläfer im hibernisierten Zustand

Die Bestrahlungen wurden mit einer 200-kV-Röntgenstrahlung $(0,5 \mathrm{~mm} \mathrm{Cu})$ bei einer Dosisleistung von $85 \mathrm{R} / \mathrm{min}$ durchgeführt. Wir begannen zunächst mit einer Dosis von $700 \mathrm{R}$. Wurden die Siebenschläfer im homoiothermen Zustand mit einer solchen Dosis bestrahlt, so entsprach die Mortalitätsrate innerhalb von 30 Tagen der von Ratten. Sie betrug in unserem Falle $63 \%$. Wurden die Tiere jedoch im Winterschlaf bestrahlt und weitere 3 Wochen im poikilothermen Zustand gehalten, so starb während dieser Zeit keines der Tiere. Erst wenn die Siebenschläfer nach dieser Zeit durch Einbringen in einen warmen Raum erwachten, traten nach einigen Tagen die typischen Symptome des akuten Strahlensyndroms auf und die Tiere starben, als ob sie zum Zeitpunkt des Erwachens bestrahlt worden wären (Abb. 5). Wenn man jedoch nach der 3 wöchigen Schlafperiode, in welcher die Strahlenschädigung zwar vorhanden sein muß, jedoch nicht zur Auswirkung kommt, weitere 30 Tage abwartet, so ist die endgültige Mortalitätsrate fast ebenso hoch wie die der im Wachzustand bestrahlten Siebenschläfer. Eine Strahlenschutzwirkung des Winterschlafs ist also offensichtlich nicht vorhanden. Es ist lediglich ein Hinauszögern der Strahlenkrankheit und des Strahlentodes, was durch den hibernisierten Zustand erreicht wird.

Die große Ahnlichkeit der beiden Überlebenskurven, die praktisch nur um den Zeitraum der Schlafperiode von 21 Tagen gegeneinander verschoben sind, brachte uns 
auf den Gedanken, den Tieren einen Strahlenschutzstoff in dem Augenblick zu injizieren, wo sie zum Zwecke des Erwachens in eine warme Umgebung gebracht werden. Gegen einen Erfolg eines solchen Vorgehens sprachen allerdings alle bislang existierenden Hypothesen ubber den Wirkungsmechanismus der Strahlenschutzsubstanzen, die ja sämtlich auf der Grundforderung basieren, daß der Schutzstoff während der Bestrahlung in den Zellen anwesend sein muß, um wirksam werden zu können. Uberraschenderweise ergab jedoch eine Versuchsserie von insgesamt 100 Tieren, die im Winterschlaf mit 700 R bestrahlt wurden, danach 21 Tage im hibernisierten Zustand ver-

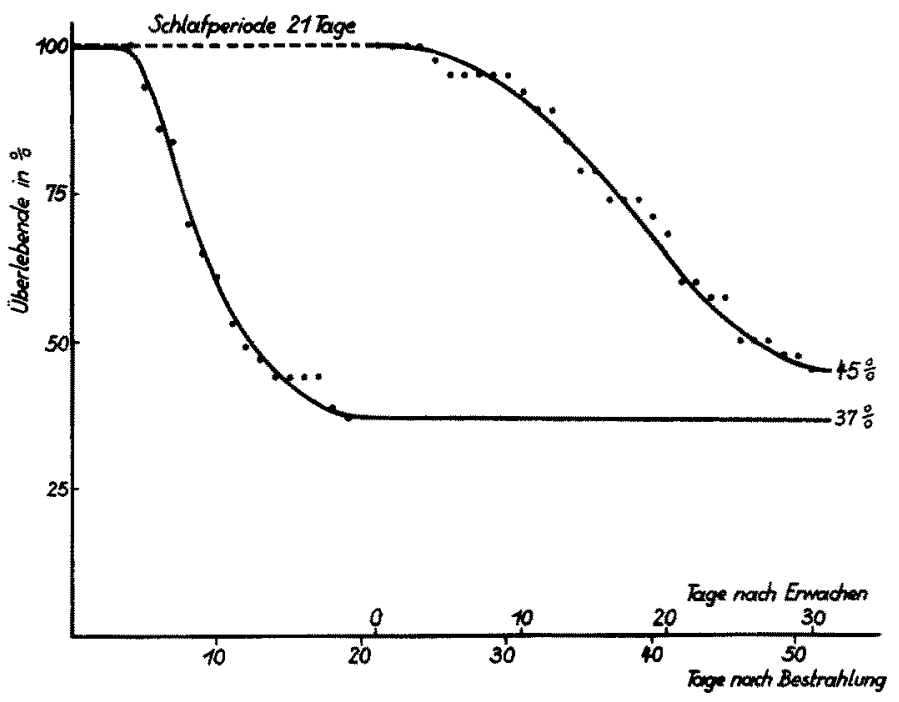

Abb. 5: Absterbeordnung wach und schlafend bestrahlter Siebenschläfer. Dosis $700 \mathrm{R}$

blieben und im Augenblick des Einbringens in eine Umgebungstemperatur von $20^{\circ} \mathrm{C}$ eine intraperitoneale Injektion von Cystein erhielten, daß nur $5 \%$ der Tiere innerhalb der folgenden 30 Tage starben. Es war bei diesem Experiment also erstmalig gelungen, durch Applikation einer radioprotektiven Substanz noch 3 Wochen $\mathrm{n} \mathrm{a} \mathrm{ch} \mathrm{der} \mathrm{Be-}$ strahlung einen signifikanten Schutzeffekt zu erzielen (Künkfl et al. 1957a, b). Bei einem weiteren Versuch mit einem anderen vielfach erprobten Schutzstoff, dem Aminoaethylisothironium, konnte allerdings ein solcher nachträglicher Schutzeffekt nicht beobachtet werden (KüNkEL, in Vorbereitung).

Diese beiden merkwürdigen Ergebnisse, die Latenz des Strahlenschadens im hibernisierten Zustand und die Möglichkeit, noch nachträglich in den Ablauf dieses Strahlenschadens durch Applikation von Cystein einzugreifen, gaben Anlaß zu weiteren Experimenten.

Zunächst ein Bestrahlungsexperiment mit höheren Dosen. Untersucht man die Abhängigkeit der Überlebenszeit ganzbestrahlter Säugetiere von der applizierten Dosis, so beobachtet man in einem Dosisbereich zwischen 1200 und $12000 \mathrm{R}$ ein eigenartiges Phänomen. In diesem Bereich ist nämlich, wie RAJEWSKY et al. (1953a, b, 1954) an Mäusen und Ratten zeigen konnten, die Überlebensdauer der Tiere nahezu unab- 
hängig von der Strahlendosis und hat den konstanten Wert von 3,5 Tagen. Auch bei anderen Säugetieren wurde ein solcher Konstanzbereich festgestellt, so z. B. bei Meerschweinchen und Affen. Uns interessierte nun, ob auch bei Siebenschläfern ein Konstanzbereich beobachtet werden kann und ob dieser Effekt auch bei hibernisierten Tieren auftritt. Die Ergebnisse dieses Versuchs sind im folgenden Diagramm dargestellt (Abb. 6). Man sieht, daß bei wachen Siebenschläfern auch ein solcher Konstanzbereich existiert, der etwa bei $3000 \mathrm{R}$ beginnt. Eine weitere Erhöhung der Dosis verkürzt die Überlebenszeit, welche hier 4,2 Tage beträgt, zunächst nicht mehr (Kurve $A$ ). Die

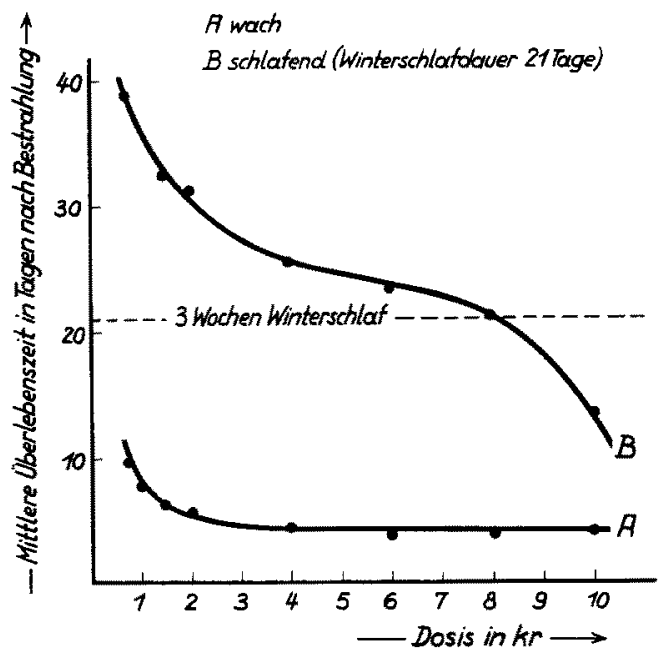

Abb. 6: Mittlere Uberlebenszeir total bestrahlter Siebenschläfer (Kurve $A$ wach, Kurve $B$ schlafend; Winterschlafdauer 21 Tage)

Kurve $B$ zeigt die Verhältnisse bei im Winterschlaf bestrahlten und 3 Wochen im hibernisierten Zustand gehaltenen Tieren. Hier stirbt bei Dosen bis zu $4000 \mathrm{R}$ während der dreiwöchigen Schlafperiode kein Tier. Bis zu dieser Dosis bleibt also der Strahlenschaden im Winterschlaf latent und wird erst nach dem Erwachen der Tiere manifest. Werden jedoch im Winterschlaf befindliche Tiere mit $8000 \mathrm{R}$ bestrahlt, so sterben bereits $25 \%$ während des hibernisierten Zustandes. Bei $10000 \mathrm{R}$ sind es bereits mehr als die Hälfte, und die mittlere Uberlebenszeit beträgt hier nur noch 13,5 Tage. Ein Konstanzbereich ist nicht mehr festzustellen (KüNKEx. 1959a).

Die nun folgenden Untersuchungen hatten zum Ziel, zunächst einmal zu prüfen, welche typischen Strahlenreaktionen bereits im Winterschlaf nachweisbar sind und welche erst nach Wiedereintritt des homoiothermen Zustandes in Erscheinung treten. Ferner sollte untersucht werden, bei welchen strahleninduzierten Reaktionen die unerwartete Schutzwirkung des Cysteins bei Applikation n a ch der Bestrahlung sichtbar wird.

So wurde zunächst mit Hilfe konventioneller cytologischer und histologischer Untersuchungsmethoden geprüft, welche Veränderungen am Knochenmark sowie einer Reihe weiterer Organe ganzkörperbestrahlter Tiere im poikilothermen und homoeo- 
thermen Zustand festgestellt werden können (KüNKEL \& HeCKManN 1958). Es ist unmöglich, im Rahmen dieses Berichtes auf die große Zahl der Einzelbefunde ausführlich einzugehen. Das wichtigste Ergebnis ist $z$ weifellos, daß strahleninduzierte Veränderungen sowohl am Knochenmark als auch an den meisten übrigen Organen bereits wäh-
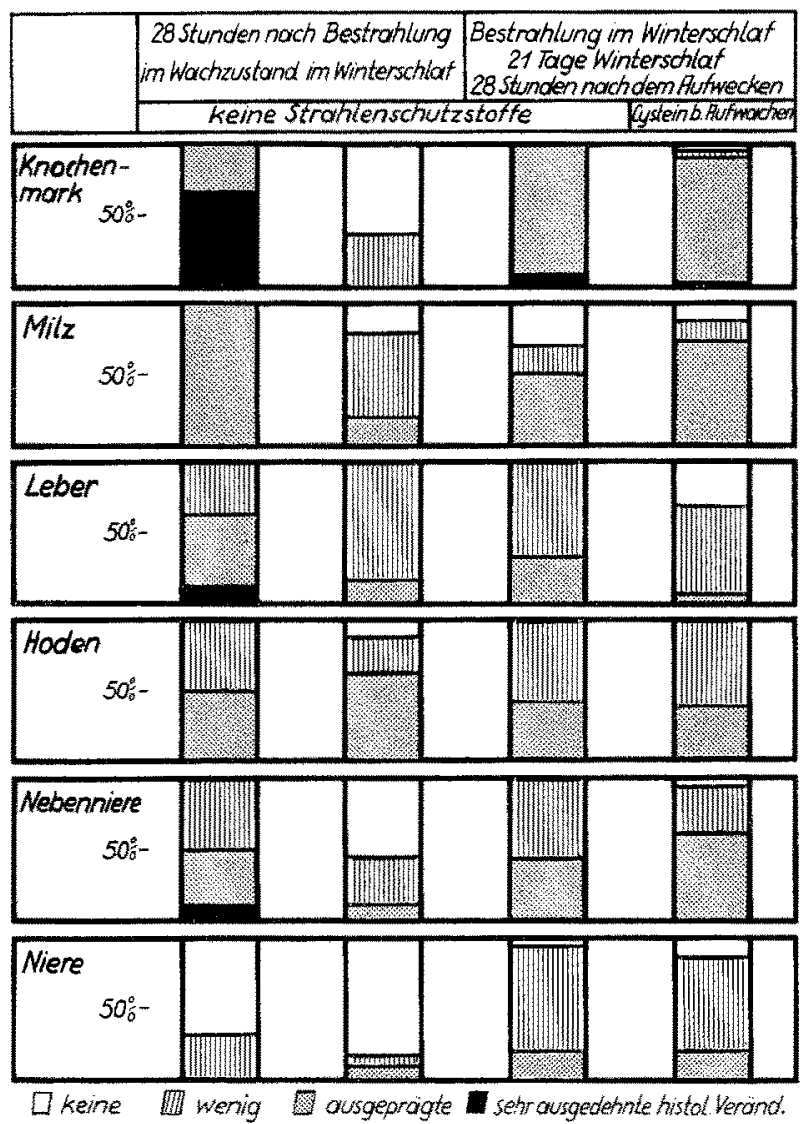

Abb. 7: Histologische Organveränderungen bei Winterschläfern nach Ganzkörperbestrahlung mit $800 \mathrm{R}$ (in $\%$ der untersuchten Tiere)

rend des Winterschlafes deutlich nachweisbar sind. Allerdings ist der Ablauf dieser Prozesse im hibernisierten Zustand außerordentlich verlangsamt. So sinkt z. B. die Gesamtzahl kernhaltiger Knochenmarkzellen während der auf die Bestrahlung folgenden 3 wöchigen Schlafperiode nur etwa halb so stark ab wie bei wachbestrahlten Tieren an einem einzigen Tag. Erst nach Uberfuhrung der Siebenschläfer in den homoiothermen Zustand vermindert sich die Zellzahl innerhalb von 24 Stunden auf fast genau den gleichen Wert, der bei den wachbestrahlten Tieren bereits 3 Wochen früher erreicht wurde. Allerdings ist ein Schutzeffekt des nach der Bestrahlung gegebenen Cysteins nur an den Plasma- und Retikulumzellen zu beobachten.

Ahnlich wie die Befunde beim Knochenmark sind auch die histologischen Verän- 
derungen bei nahezu allen untersuchten Organen (Abb. 7). Gerade bei den histologischen Untersuchungen zeigt sich deutlich, daß bei den im Winterschlaf bestrahlten Tieren die Entwicklung des Strahlenschadens infolge des verlangsamten Ablaufes wie bei einer Zeitlupenaufnahme beobachtet werden kann. Da eine Wiedergabe der zahlreichen histologischen Bilder hier nicht möglich ist, wurden die Ergebnisse in einem Schaubild zusammengestellt. Dabei sind die Organe in der Reihenfolge von oben nach

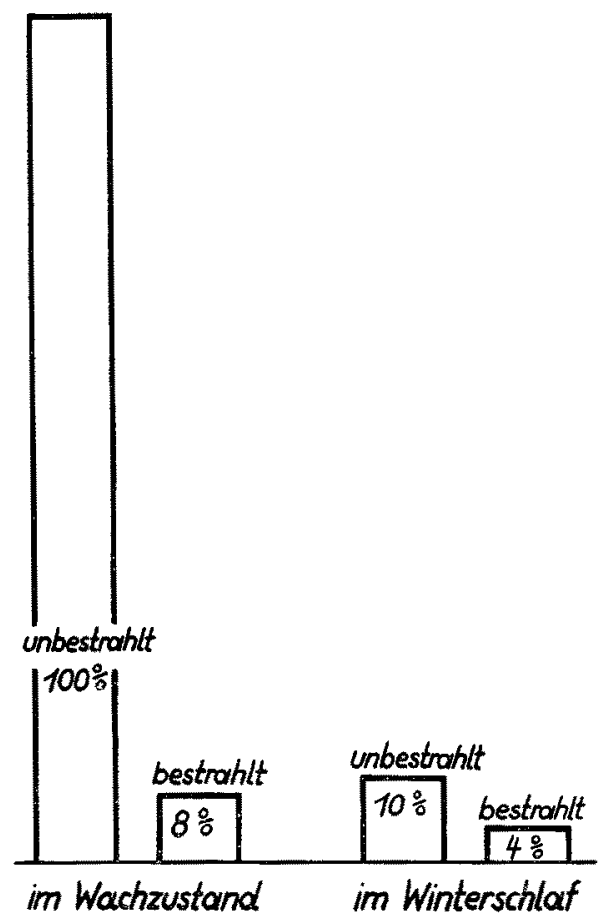

Abb. 8: Einbau von radioaktiv markiertem Eisen in die Erythrozyten von Siebenschläfern

unten nach steigender Strahlenresistenz geordnet. Die senkrechten Spalten zeigen 4 verschiedene Versuchsgruppen. Wie aus der Abbildung zu ersehen ist, weicht die Leber auffallend von den übrigen Organen $a b$. Sie ist, abgesehen von den oben erwähnten Plasma- und Retikulumzelien des Knochenmarks, das einzige Organ, bei welchem ein deutlicher Schutzeffekt des Cysteins bei Applikation nach der Bestrahlung erkennbar ist.

Eine weitere typische Strahlenreaktion, welche in einem engen Zusammenhang mit der gestörten Zellneubildung im Knochenmark steht, ist die Hemmung des Eiseneinbaues in die Erythrozyten. Auch dieser strahlenbiologische Test wurde von uns bei wachen und hibernisierten Siebenschläfern mit Hilfe von radioaktivem Eisen geprüft (KüNKEL 1959b). Dieses ist bekanntlich eine sehr strahlenempfindliche Reaktion, deren Hemmung bereits relativ frühzeitig nach der Strahleneinwirkung gemessen werden kann. Die Ergebnisse sind in Abbildung 8 dargestellt. Setzt man die Einbaurate bei den unbestrahlten homoiothermen Kontrolltieren gleich $100 \%$, so ergibt sich 8 Tage nach Bestrahlung mit $800 \mathrm{R}$ eine um $92 \%$ erniedrigte Aktivität der Erythrozyten. Im 
hibernisierten Zustand beträgt die Einbaurate bei unbestrahlten Tieren nur noch $10 \%$, entsprechend der im Winterschlaf stark reduzierten Zellneubildung. Doch ist auch hier nach der Bestrahlung eine weitere Hemmung um $60 \%$ festzustellen, so daß die Einbaurate nur noch $4 \%$ von derjenigen beträgt, die bei den wachen unbestrahlten Kontrollen nachgewiesen wurde.

Ebenfalls am peripheren Blut, munmehr jedoch am Serum, wurden die folgenden Untersuchungen durchgeführt (SCHUBERT, KüNKEL \& MAAss 1957). Wie wir bereits vor 12 Jahren an ganzkörperbestrahlten Ratten zeigen konnten, verändert sich etwa vom 3. bis 4. Tag nach der Bestrahlung an die Eiweißzusammensetzung des Serums (HÖHNE, JASTER \& KüNKEL 1952). Der relative Anteil der Albumine und der Gammaglobuline am Gesamteiweiß sinkt ab, Alpha- und Betaglobuline nehmen zu. Diese Ver-

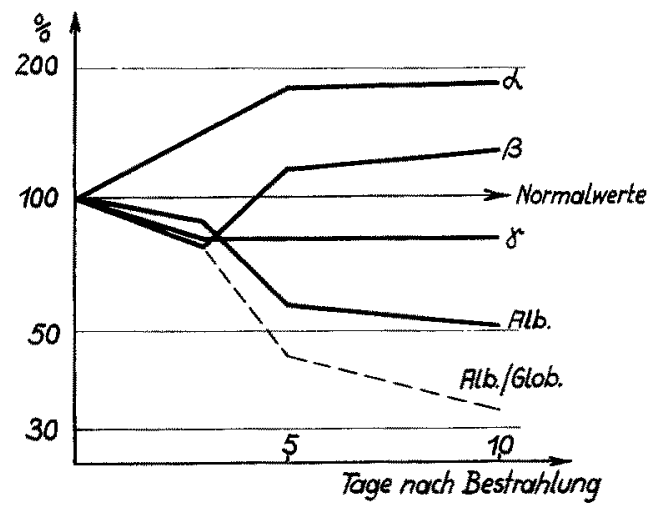

Abb. 9: Veränderung der Serum-Eiweißzusammensetzung von Siebenschläfern nach Bestrahlung im Wadzustand

schiebungen erreichen ihr Maximum zwischen dem 6. und 10. Tag nach der Bestrahlung. Das gleiche Bild wie bei Ratten sieht man auch bei Siebenschläfern. Untersucht man die unbestrahlten Siebenschläfer im Wachzustand, im hibernisierten Zustand und nach dem Erwachen, so erkennt man bereits bei diesen unbehandelten Tieren Unterschiede im Serumeiweißspektrum. Sie entsprechen den jahreszeitlichen Schwankungen, die auch SUOMALAINEN (1953) an wachen und hibernisierten Igeln festgestellt hat. So ist z. B. das Albumin-Globulin-Verhältnis bei wachen Siebenschläfern mit 0,9 am niedrigsten, beträgt im Winterschlaf ca. 1,8, um nach dem Erwachen allmählich wieder abzusinken. Die Ergebnisse der Bestrahlungsexperimente sind in Abbildung 9 dargestellt. Setzt man die Normalwerte der einzelnen Serumeiweißfraktionen unbestrahlter, wacher Tiere gleich 100 , so ergeben sich die typischen Verschiebungen, die auch bei Ratten, Mäusen und anderen Säugetieren beobachtet werden. Nach Totalbestrahlung im Winterschlaf sind jedoch gegenüuber den Normalwerten hibernisierter Tiere Verschiebungen in der Serumeiweißzusammensetzung praktisch kaum nachweisbar, solange die Tiere in diesem Zustand verbleiben. Werden sie jedoch entsprechend der bei allen diesen Experimenten gleichen Versuchsanordnung nach drei Wochen wieder in den homoiothermen Zustand versetzt, so treten etwa 4 bis 5 Tage nach dem Erwachen die typischen strahleninduzierten Veränderungen auf (Abb. 10). Eine intra- 
peritoneale Injektion von Cystein zum Zeitpunkt des Erwachens hat jedoch eine stark reduzierende Wirkung auf die Ausbildung dieser Strahlenreaktion, wie Abbildung 11 erkennen läßt.

Für den Biochemiker wie auch für den Strahlenbiologen besonders interessant ist stets die Desoxyribonukleinsäure. Die Hemmung der Synthese der DNS ist zweifellos eine der empfindlichsten biologischen Strahlenreaktionen überhaupt. Schon nach relativ kleinen Strahlendosen läßt sich diese Hemmung bereits wenige Minuten nach der Be-

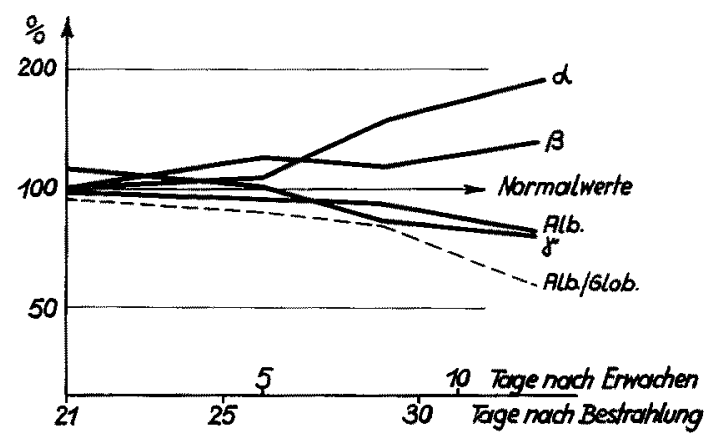

Abb. 10: Veränderung der Serum-Eiweißzusammensetzung von im Winterschlaf bestrahlten Siebenschläfern nach dem Erwachen

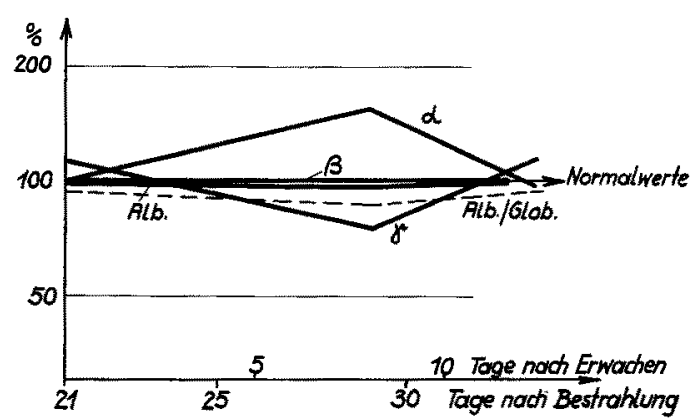

Abb. 11: Veränderung der Eiweißzusammenserzung von im Winterschlaf bestrahlten Siebenschläfern nach Cystein-Applikation beim Erwachen

strahlung einwandfrei feststellen (ORD \& STOCKEN 1957). In unseren Versuchen wurde der Einbau von Radiophosphor in die DNS der Darmepithelzellen der Siebenschläfer gemessen (KüNkel 1958, KüNKel \& SchUbERT 1958). Die Ergebnisse sind in der Tabelle 1 zusammengestellt.

Die Dosis betrug hier 800 R. Bei den im Wadhzustand bestrahlten Tieren (Gruppe 2) ergibt sich unter den erwähnten Bedingungen eine Hemmung um rund $50 \%$ gegenüber den unbestrahlten Kontrollen (Gruppe 1). Wegen der im Winterschlaf stark reduzierten Zellteilungsaktivität ist bei den winterschlafenden Tieren der Gruppe 3 die Einbaurate weniger als halb so hoch wie im Wachzustand. Jedoch ist auch hier 24 Stunden nach Bestrahlung im Winterschlaf eine starke Hemmung des ${ }^{32} \mathrm{P}$-Einbaues in die DNS festzustellen. Die DNS-Synthese bei den Tieren der Gruppe 5 ist 24 Stunden 
Tabelle 1

DNS-Synthese im Darm von Siebenschläfern

\begin{tabular}{|c|c|c|c|c|c|}
\hline Gruppe & $\begin{array}{l}\text { Zahld. } \\
\text { Tiere }\end{array}$ & Behandlung & $\begin{array}{c}\text { Dauer des } \\
\text { Winterschlafes }\end{array}$ & $\begin{array}{l}\text { Zeitpunkt der } \\
\text { 32P-Injektion }\end{array}$ & $\begin{array}{l}\text { Einbau- } \\
\text { rate von } \\
\text { 32p }\end{array}$ \\
\hline $\begin{array}{l}1 \\
2\end{array}$ & $\begin{array}{l}18 \\
18\end{array}$ & $\left.\begin{array}{l}\text { Kontrollen } \\
\text { Bestrahlung }\end{array}\right\}_{\text {zustand }}^{\text {im Wach- }}$ & - & $\begin{array}{l}\text { im Wachzustand } \\
\text { im Wachzustand } \\
\text { (24 Std. nach } \\
\text { Bestrahlung) }\end{array}$ & $\begin{array}{l}63 \\
32\end{array}$ \\
\hline $\begin{array}{l}3 \\
4\end{array}$ & $\begin{array}{l}18 \\
18\end{array}$ & $\begin{array}{l}\text { Kontrollen } \\
\text { Bestrahlung }\end{array}$ & $\begin{array}{l}\text { während des gan- } \\
\text { zen Versuchs }\end{array}$ & $\begin{array}{l}\text { im Winterschlaf } \\
\text { im Winterschlaf } \\
\text { (24 Std. nach } \\
\text { Bestrahlung) }\end{array}$ & $\begin{array}{l}29 \\
11\end{array}$ \\
\hline $\begin{array}{l}5 \\
6 \\
7\end{array}$ & $\begin{array}{l}16 \\
18 \\
14\end{array}$ & \begin{tabular}{l|l} 
Kontrollen & im Win- \\
Bestrahlung & im Win- \\
Bestrahlung & terschlaf \\
u. Cystein- & \\
injektion \\
21 Tage nach \\
Bestrahlung
\end{tabular} & $\begin{array}{l}21 \text { Tage } \\
21 \text { Tage } \\
21 \text { Tage }\end{array}$ & $\begin{array}{l}24 \text { Std. nach } \\
\text { Rückkehr in den } \\
\text { Wachzustand }\end{array}$ & $\begin{array}{l}47 \\
87 \\
47\end{array}$ \\
\hline
\end{tabular}

nach Rückkehr in den homoiothermen Zustand noch nicht ganz so intensiv wie bei den unbestrahlten wachen Kontrollen. Bei den im Winterschlaf bestrahlten Siebenschläfern der Gruppe 6 ist 24 Stunden nach Erwachen, also 22 Tage nach der Bestrahlung die DNS-Synthese eigenartigerweise fast doppelt so hoch wie bei den Tieren der Gruppe 1. Der Schutzeffekt des Cysteins bei den Tieren der Gruppe 7 ist eindeutig. Die Einbauraten liegen hier in der gleichen Höhe wie bei den unbestrahlten Siebenschläfern.

Wegen der Kürze der Zeit möchte ich nur am Rande erwähnen, daß wir auch die stationären. Konzentrationen der Adeninnukleotide in den Leberzellen bestimmt haben (KüNKEL 1961). Obwohl wir erwartet hatten, daß bei dem verminderten Energiebedarf der Zellen im Winterschlaf auch ein vermindertes Angebot an energiereichen Phosphaten bestehen würde, zeigte sich zu unserer Überraschung, daß z. B. die Konzentration des ATP im Winterschlaf erheblich erhöht ist. Ein weiterer Versuch über die Entspeicherung des neurosekretorischen Systems im Hypophysen-Hinterlappen ist zur Zeit noch in der Auswertung.

Zusammenfassend kann festgestellt werden, daß strahleninduzierte Reaktionen, die im homoiothermen Zustand erst einige Tage nach der Bestrahlung in Erscheinung treten, im Winterschlaf nicht festgestellt werden können. Typische Frühreaktionen auf Ganzkörperbestrahlungen sind jedoch auch schon im hibernisierten Zustand, wenn auch nicht so stark, so doch deutlich ausgeprägt.

Trotz der Vielzahl der bei diesen Versuchen erhaltenen Einzelergebnisse sind wir von einer Klärung der diesen merkwürdigen Phänomenen zugrunde liegenden Mechanismen noch weit entfernt. Ein Bild, das diese Phänomene zwar nicht erklärt, aber doch recht anschaulich beschreibt, vergleicht die primäre Strahlenschädigung mit der Verunreinigung eines empfindlichen Motors mit Sandkörnern. Befindet sich die Maschine in Ruhe, so bleibt der Schaden unbemerkt. Erst während des Betriebes führen diese 
"Sandkörner" zum Versagen, und zwar um so früher, je schneller die Maschine läuft und je größer die Beanspruchung ist. Ob langlebige strahleninduzierte paramagnetische Zentren, wie sie von ZrMmer et al. (1957) an ruhenden Systemen wie kristallisierten Aminosäuren oder trockenen Pflanzensamen nach Bestrahlung mit Hilfe der Mikrowellenspektroskopie gefunden wurden, eine Rolle spielen, konnte bisher noch nicht nachgeprüft werden. Ganz ohne Zweifel ist jedoch die Ausprägung und Manifestierung des Strahlenschadens unmittelbar von der jeweiligen Stoffwechselaktivität des betreffenden Objektes abhängig.

\section{ZUSAMMENFASSUNG}

1. Werden Siebenschläfer im hibernisierten Zustand mit einer semiletalen Dosis von Röntgenstrahlen bestrahlt, so bleibt der Strahlenschaden mehrere Wochen latent, falls die Tiere während dieser Zeit im Winterschlaf verbleiben. Er wird erst nach dem Erwachen der Tiere manifest. Die Tiere sterben dann, als ob sie zum Zeitpunkt des Erwachens bestrahlt worden wären.

2. Die endgültige Mortalitätsrate ist nicht wesentlich von der im Wachzustand bestrahlter Tiere verschieden. Der Winterschlaf an sich bewirkt also lediglich ein Hinauszögern des Strahlentodes, jedoch keinen Strahlenschutz.

3. Werden im Winterschlaf bestrahlte Winterschläfer 3 Wochen im hibernisierten Zustand gehalten, und danach zum Zeitpunkt der Uberführung in den homoiothermen Zustand mit Cystein behandelt (500 $\mathrm{mg} / \mathrm{kg}$ i. p.), so wird noch nachträglich ein signifikanter Strahlenschutz erzielt.

4. Bei wachen Siebenschläfern existiert $z$ wischen 3000 und 10000 R ein Konstanzbereich der Uberlebenszeit von 4,2 Tagen (RAJEWSKY-Effekt), bei hibernisierten Tieren jedoch nicht.

5. Strahleninduzierte, morphologische Veränderungen sind bereits im Winterschlaf sowohl am Knochenmark als auch an einigen anderen Organen nachweisbar, wenn auch wesentlich schwächer ausgeprägt als im Wachzustand.

6. Die Erythrozytenproduktion beträgt im Winterschlaf nur $8 \%$ von der im Wachzustand beobachteten (Radioeisentest). Die strahleninduzierte Hemmung ist auch im Winterschlaf erheblich.

7. Die im Wachzustand auftretenden strahleninduzierten Veränderungen der Serumeiweißzusammensetzung können im Winterschlaf nicht beobachtet werden. Sie sind erst nach dem Wiedererwachen der Tiere nachweisbar.

8. Die DNS-Synthese der Darmepithelzellen ist im Winterschlaf um ca. $50 \%$ vermindert. Eine strahleninduzierte Hemmung um weitere $60 \%$ ist jedoch nachweisbar.

9. ATP ist im Winterschlaf erhöht.

10. Die Erythrozytenlebensdauer ist im Winterschlaf auf etwa das 3 fache verlängert. 


\section{ZITIERTE LITERATUR}

HÖHNE, G., JASTER, R. \& KÜNKEL, H. A., 1952. Elektrophoretische Untersuchungen am Serum röntgenbestrahlter Ratten. Klin. Wschr. 30, 952.

KüNKEL, H. A., 1958. Die Beeinflußbarkeit der strahleninduzierten Hemmung der DNS-Syn“ these durch Cystein bei normalem und reduziertem Stoffwechsel. Strablenther. Sdbd 38, $17-22$.

- 1959a. Zur Wirkung holner Strahlendosen bei Siebenschläfern. Z. Naturf. 14b, 757-759.

- 1959b. Uber den Einbau von Radioeisen in die Erythrozyten röntgenbestrahlter Siebenschläfer. Naturwissenschaften 46, 627-628.

- 1961. Strahlenbiologische Untersuchungen an hibernisierten Siebenschläfern. Chemotberapia $3,200-224$.

- \& Hzckmann, U., 1958. Die Strahlenschutzwirkung von Cystein und Cysteamin bei reduziertem Stoff wechsel. Strablenther, 106, 256-259.

- Höhne, G. \& MaAss, H., 1957a. Der Einfluß von Cystein und Winterschlaf auf die Uberlebensrate röntgenbestrahlter Siebenschläfer (Glis glis). Z. Naturf. 12b, 144-147.

-, -, - 1957b. Radiobiological investigations on the hibernating loir (Glis glis). In: „Advances in Radiobiology“, Oliver \& Boyd, London, pp. 176-179.

- \& Schubert, G., 1958. The influence of total-body irradiation on deoxyribonucleic acid synthesis and the protective action of cysteine. (Investigations on rats and hibernating loirs.) Radiation Res. 9, 141.

-, - 1959. Effects of protective agents applied after irradiation. In: "Progress in Nuclear

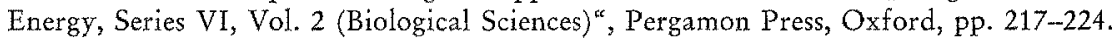

Ord, M. G. \& STOcken, L. A., 1957. The effect of X-radiation on rat thymus nucleic acids at short intervals after exposure in vivo. In: "Advances in Radiobiology", Oliver \& Boyd, London.

Rajewsky, B., Aurand, K. \& Heuse, O., 1953. Weitere Untersuchungen zum Problem der Bestrahlung der weißen Maus mit hohen Dosen von Röntgenstrahlen. Z. Naturf. $8 \mathbf{b}$, $524-526$.

-, -, - 1953. Weitere Untersuchungen zum Problem der Ganzkörper-Bestrahlung der weißen Maus. Sofortiger Tod durch Strahlung. Z. Naturf. 8b, 157-159.

,,---1954 . Bestrahlungen von weißen Mäusen mit hohen Dosen von Röntgenstrahlen. Strablenther. 95, 513-522.

SCriubert, G., KüNEEL, H. A. \& MaAss, H., 1957. Elektrophoretische Untersuchungen am Serum röntgenbestrahlter und hibernisierter Siebenschläfer (Glis glis). Strablenther. 103, 368-375.

Suomalainen, P. \& Leskinen, E., 1953. Suomen Kemistilehti B 2, 15-16.

Zimmer, K. G., EhrenberG, L. \& EhrenberG, A., 1957. Nachweis langlebiger magnetischer Zentren in bestrahlten biologischen Medien und deren Bedeutung für die Strahlenbiologie. Strablenther. 103, 3-15.

\section{Diskussion im Anschluß an den Vortrag KüNKEL}

YON BERTALANFFY: You have found, as a somewhat paradoxical effect of non-lethal irradiation, an increase of DNA synthesis. A similar effect - initial increase followed by decrease - is known for RNA after irradiation (e. g. von Bertalanfry 1963. Protoplasma 57, 51-83; 1959. Acta Cytol. 3, 367). The causes are unknown. Do you have any explanation?

KüNKEL: Eine Erklärung habe ich nicht dafür. Es wäre denkbar, daß ein abbauendes Ferment geschädigt worden ist.

Horstmann: Haben Sie versucht, die Siebenschläfer $n a c h$ der Bestrahlung in den Winterschlaf zu bringen? Wie verhalten sie sich dabei?

KüNkEL: Ja, das haben wir versucht, und es hat sich dabei herausgestellt, daß die Tiere dann bereits während des Winterschlafes absterben. Die Entwicklung des Strahlenschadens geht 
offenbar so schnell vor sich, daß die kritische Schwelle bereits beim Ubergang in den Winterschlaf erreicht ist. Bis die Tiere fest eingeschlafen sind, dauert ja ohnehin mindestens einen Tag. Noch eine andere interessante Tatsache: Wir können die Siebenschläfer ja nicht züchten, sondern müssen sie fangen lassen. Die frischgefangenen Tiere sind außerordentlich wild und können nur mit dicken Motorrad-Handschuhen angefaßt werden. Sie haben sehr spitze Zähne und springen 2 bis $3 \mathrm{~m}$ durch die Luft, sind also recht gefährlich. Wenn diese Tiere aber einige Wochen lang im. Winterschlaf waren, dann sind sie die zahmsten Tiere der Welt. Sie haben also offenbar ihre natürliche Wildheit im. Winterschlaf vergessen.

KrÜGer: Aus den Ausführungen von Herrn Heusner scheint hervorzugehen, daß̧ während des Winterschlafes der Aufbau-Stoffwechsel zumindest reduziert ist, während der AbbauStoff wechsel weiterläuft. Wenn wir annehmen, daß die Strahlung nur den Aufbau-Stoffwechsel schädigt, nicht aber den Abbau-Stoffwedhsel, läßt sich deuten, daß während des Winterschlafes die Strahlenschädigungen nicht manifest werden.

KüNKEL: Ja, so etwas wird sicherlich eine Rolle dabei spielen. Allerdings bleibt der AbbauStoff wechsel nicht in allen Fällen konstant. So werden z. B. die Erythrozyten im Winterschlaf sehr viel langsamer abgebaut als im wachen Zustand. Andererseits ist ein Strahleneinfluß auf die Erythrozytenlebensdauer nicht erkennbar. 